\title{
Secondary Raynaud Phenomenon
}

National Cancer Institute

\section{Source}

National Cancer Institute. Secondary Raynaud Phenomenon. NCI Thesaurus. Code

C116359.

Raynaud phenomenon associated with an underlying autoimmune disorder. 